Some Computational Insights on the Optimal Bus Transit Route Network Design Problem Author(s): Wei (David) Fan, Randy B. Machemehl, and Nicholas E. Lownes

Source: Journal of the Transportation Research Forum, Vol. 47, No. 3 (Public Transit Special Issue 2008), pp. 61-75

Published by: Transportation Research Forum

Stable URL: http://www.trforum.org/journal

The Transportation Research Forum, founded in 1958, is an independent, nonprofit organization of transportation professionals who conduct, use, and benefit from research. Its purpose is to provide an impartial meeting ground for carriers, shippers, government officials, consultants, university researchers, suppliers, and others seeking exchange of information and ideas related to both passenger and freight transportation. More information on the Transportation Research Forum can be found on the Web at www.trforum.org. 


\title{
Some Computational Insights on the Optimal Bus Transit Route Network Design Problem
}

\author{
by Wei (David) Fan, Randy B. Machemehl, and Nicholas E. Lownes
}

The objective of this paper is to present some computational insights based on previous extensive research experiences on the optimal bus transit route network design problem (BTRNDP) with zonal demand aggregation and variable transit demand. A multi-objective, nonlinear mixed integer model is developed. A general meta-heuristics-based solution methodology is proposed. Genetic algorithms (GA), simulated annealing (SA), and a combination of the GA and SA are implemented and compared to solve the BTRNDP. Computational results show that zonal demand aggregation is necessary and combining metaheuristic algorithms to solve the large scale BTRNDP is very promising.

\section{INTRODUCTION}

Public transit has been widely recognized as a means of reducing air pollution, lowering energy consumption, improving mobility and lessening traffic congestion (Bailey 2007). Designing an operationally and economically efficient bus transit network is very important for the urban area's social, economic and physical structure.

In the past decade, several research efforts have examined the bus transit route network design problem (BTRNDP). It's well understood that several sources of complexity, including nonconvexities, non-linearities, the inherent multi-objective cost characteristics, the combinatorial complexity due to the discrete BTRNDP nature and the huge candidate solution set, often render the solution search space computationally intractable. This computational burden grows exponentially with the size of the bus transit network (Baaj and Mahmassani 1991).

Previous approaches used to solve the BTRNDP can be classified into three categories: 1) Practical guidelines and ad hoc procedures; 2) Analytical optimization models for idealized situations; and 3) Meta-heuristic approaches for more practical problems. In the early stages of the research on bus transit route network design problems, traditional operations research analytical optimization models were used. Rather than determine both the route structure and design parameters simultaneously, these analytical optimization models were primarily applied to determine one or several design parameters (e.g., stop spacing, route spacing, route length, service coverage, bus size and/or frequency of service) on a predetermined transit route network structure (Newell 1979, Leblanc 1988, Chang and Schonfeld, 1991 and 1993, Chien and Schonfeld 1997, Spasovic et al. 1993, and Spasovic and Schonfeld 1994). NCHRP Synthesis of Highway Practice 69 provides industry rules-of-thumb service planning guidelines based largely on these analytic methods.

Generally speaking, these analytical optimization models are very effective in solving optimization-related problems for networks of small size or with one or two decision variables. However, when it comes to the transit route design problem for a network of realistic size in which many parameters need to be determined, this approach does not work very well. Due to the inherent complexity involved in the BTRNDP, meta-heuristic approaches that pursue reasonably good local optima but do not guarantee the global optimal solution were proposed.

The heuristic (Lampkin and Saalmans 1967, Rea 1971, Silman et al. 1974, Dubois et al. 1979, Axhausemm and Smith 1984, Ceder and Wilson 1986, Ceder and Israeli 1998, Van Nes et al. 1988, Baaj and Mahmassani 1991, Shih et al. 1998, Lee and Vuchic 2000, and Zhao and Zeng 2007) and meta-heuristic approaches primarily dealt with simultaneous design of the transit route network and determination of its associated bus frequencies. Those meta-heuristics methods used for solving 
the BTRNDP with fixed transit demand include genetic algorithms (Pattnaik et al. 1998, Chien et al. 2001, Chakroborty and Dwivedi 2002, Bielli et al. 2002, Chakroborty 2003, Fan 2004, and Fan and Machemehl 2006a), simulated annealing (Fan 2004, and Fan and Machemehl 2006b), and tabu search (Fan 2004, and Fan and Machemehl 2004). To make the BTRNDP more tractable, all previous research work assumed bus transit demand as fixed. This approach is problematic because the bus transit demand actually depends on the specific configuration of the bus transit network structure and its route frequencies. This variable relationship existing between the transit demand and network configuration makes the consideration of the BTRNDP with variable transit demand a necessity. Limited research on the BTRNDP with variable transit demand can provide some insights (Lee and Vuchic 2000, Fan 2004, and Fan and Machemehl 2006a), though research efforts have used one algorithm alone to solve the BTRNDP. It is believed that the warm start method (using the solutions obtained from one algorithm as a starting point for another algorithm) will have superior performance compared to using a single algorithm alone.

As such, the objective of this paper is to present computational insights based on extensive research experience on the BTRNDP with variable (elastic) transit demand. The main original contribution of this paper is to emphasize the computational necessity of zonal demand aggregation, introduce the warm start concepts, and combine and compare two very advanced meta-heuristics methods to solve the BTRNDP for the first time. In particular, Genetic algorithms (GA), simulated annealing algorithm (SA), and a combination of the GA and SA are implemented to solve the BTRNDP. Particular attention is given to the algorithm comparisons and warm start component.

The subsequent sections of this paper are organized as follows: section 2 presents model formulation of the BTRNDP. Section 3 proposes the solution methodology for the BTRNDP with variable transit demand. Section 4 discusses the comprehensive computational results of the experimental network. Finally, a summary and discussion of future research directions concludes this paper in section 5 .

\section{MODEL FORMULATION}

The BTRNDP transportation system can be described in terms of "nodes," "links" and "routes." Consider a connected network with a directed graph $\mathrm{G}=\{N, A\}$ consisting of a finite set of $N$ nodes and $A$ links (arcs) which connect pairs of nodes with the following relevant notation.

\section{Sets/Indices:}

$i, j \in N$

$r_{k} \in R$

Centroid nodes $i$ and $j$ (i.e., zones)

$i_{t} \in N$

$k$-th route

tr $\subset R$

$t$-th distribution node of centroid node $i$

transfer paths that use more than one route from $\mathrm{R}$

\section{Data:}

$\mathrm{R}_{\text {max }}=$ maximum allowed number of routes for the route network

$\mathrm{N}=$ number of centroid nodes in the route network

$\mathrm{D}_{\text {max }}=$ maximum length of any route in the transit network

$\mathrm{D}_{\text {min }}=$ minimum length of any route in the transit network

$\mathrm{d}_{\mathrm{ij}}=$ total demand between centroid nodes $i$ and $j$

$\mathrm{h}_{\max }=$ maximum headway required for any route

$\mathrm{h}_{\text {min }}=$ minimum headway required for any route 
$\mathrm{L}_{\max }=$ maximum load factor for any route

$V_{b}=$ the bus travel speed

$\mathrm{P}=$ seating capacity of buses operating on the network

$\mathrm{F}=$ maximum bus fleet size available for operations on the route network

$\mathrm{C}_{\mathrm{v}}=$ per-hour operating cost of a bus; (\$/vehicle/hour)

$\mathrm{C}_{\mathrm{m}}=$ value of time $(\$ / \mathrm{min})$

$\mathrm{O}_{\mathrm{v}}=$ operating hours for the bus running on any route; (hours)

$\mathrm{C}_{\mathrm{d}}=$ value of each unsatisfied travel demand in dollars (\$/person)

$\mathrm{W}_{1}, \mathrm{~W}_{2}, \mathrm{~W}_{3}=$ weights reflecting the relative importance of three components: the user costs, operator costs and unsatisfied total demand costs respectively

\section{Decision Variables:}

$M=$ the number of routes of the current proposed bus transit network solution

$r_{m}=$ the $m$-th route of the proposed solution, $m=1,2, \ldots, M$

$D_{r_{m}}=$ the overall length of route $r_{m}$

$d_{i j}^{r_{m}}=$ the bus transit travel demand between centroid nodes $i$ and $j$ on route $r_{m}$

$d_{i j}^{t r}=$ the bus transit travel demand between centroid nodes $i$ and $j$ along transfer path $t r$

$D R_{i j}=$ the set of direct routes used to serve the demand from centroid nodes $i$ and $j$

$T R_{i j}=$ the set of transfer paths used to serve the demand from centroid nodes $i$ and $j$

$t_{i j}^{r_{m}}=$ the total travel time between centroid node $i$ and $j$ on route $r_{m}$

$t_{i j}^{t r}=$ the total travel time between centroid node $i$ and $j$ along transfer path $t r$

$h_{r_{m}}=$ the bus headway operating on route $r_{m}$ (hours/vehicle)

$L_{r_{m}}=$ loading factor in route $r_{m}$

$T_{r_{m}}=$ the round trip time of route $r_{m} ; T_{r_{m}}=2 D_{r_{m}} / V_{b}$

$N_{r_{m}}=$ the number of operating buses required on route $r_{m} ; \mathrm{N}_{r_{m}}=T_{r_{m}} / h_{r_{m}}$

$Q_{r_{m}}^{\max }=$ the maximum flow occurring on the route $r_{m}$ (person/hour)

\section{Objective Function:}

The objective is to minimize the sum of user cost, operator cost and unsatisfied demand costs for the studied bus transit network. The objective function is as follows:

$$
\begin{aligned}
\min z= & \mathrm{W}_{1} *\left(\sum_{i \in N} \sum_{j \in N} \sum_{r_{m} \in D R_{i j}} d_{i j}^{r_{m}} t_{i j}^{r_{m}}+\sum_{i \in N} \sum_{j \in N} \sum_{t r \in T R_{i j}} d_{i j}^{t r} t_{i j}^{t r}\right)+\mathrm{W}_{2} * \frac{\mathrm{C}_{\mathrm{v}}}{\mathrm{C}_{\mathrm{m}}} * \mathrm{O}_{\mathrm{v}} *\left(\sum_{m=1}^{\mathrm{M}} \frac{T_{r_{m}}}{h_{r_{m}}}\right) \\
& +\mathrm{W}_{3} * \frac{\mathrm{C}_{\mathrm{d}}}{\mathrm{C}_{\mathrm{m}}} *\left(\sum_{i \in N} \sum_{j \in N} d_{i j}-\sum_{i \in N} \sum_{j \in N} \sum_{r_{m} \in D R_{i j}} d_{i j}^{r_{m}}-\sum_{i \in N} \sum_{j \in N} \sum_{t r \in T R_{i j}} d_{i j}^{t r}\right)
\end{aligned}
$$


s.t.

$$
\begin{array}{lll}
\mathrm{h}_{\min } \leq h_{r_{m}} \leq \mathrm{h}_{\max } & r_{m} \in \mathrm{R} & \text { (headway feasibility constraint) } \\
L_{r_{m}}=\frac{Q_{r_{m}}^{\max } * h_{r_{m}}}{\mathrm{P}} \leq \mathrm{L}_{\max } & r_{m} \in \mathrm{R} & \text { (load factor constraint) } \\
\sum_{m=1}^{\mathrm{M}} N_{r_{m}}=\sum_{m=1}^{\mathrm{M}} \frac{T_{r_{m}}}{h_{r_{m}}} \leq \mathrm{F} & r_{m} \in \mathrm{R} & \text { (fleet size constraint) } \\
\mathrm{D}_{\min } \leq D_{r_{m}} \leq \mathrm{D}_{\max } & r_{m} \in \mathrm{R} & \text { (trip length constraint) } \\
M \leq \mathrm{R}_{\max } & & \text { (maximum numbers of routes constraint) }
\end{array}
$$

The first term of the objective function is the total user cost (including the user cost on direct routes and on transfer paths), the second is the total operator cost, and the third component is the cost resulting from total travel demand, excluding the transit demand satisfied by a specific network configuration. Note that $\mathrm{W}_{1}, \mathrm{~W}_{2}$ and $\mathrm{W}_{3}$ are introduced to reflect the tradeoffs between the user costs, the operator costs and unsatisfied travel trips, making BTRNDP a multi-objective optimization problem.

Generally, operator cost refers to the cost of operating the required buses. User costs usually consist of four components: walking cost, waiting time, transfer cost, and in-vehicle cost (i.e., invehicle time required for riding a bus to a destination). Generally, these three weights are dependent on the planners' experience and expert judgment. Different settings for these weights might result in different optimal transit route networks using the developed solution methodology. However, once a specific weight set is chosen for the user costs, the operator costs and unsatisfied travel trips costs, an optimal transit route network can be identified.

For simplicity, the model assumes uniform bus size across the fleet. The first constraint is the headway feasibility constraint, which reflects the necessary usage of policy headways in extreme situations. The second is the load factor constraint, which guarantees that the maximum flow on the critical link of any route $r_{\mathrm{m}}$ cannot exceed the bus capacity on that route. The third (fleet size) constraint represents the resource limits of the transit company and guarantees that the optimal network pattern never uses more vehicles than the currently available ones. The fourth constraint is the trip length constraint. This avoids routes that are exceptionally long - bus schedules on very long routes are difficult to maintain. Conversely, to guarantee the efficiency of the network, the length of routes should not be too short. The fifth constraint sets the maximum number of routes, which reflects the fact that transit planners often set a maximum number of routes based on fleet size; this will have a great impact on driver scheduling.

\section{PROPOSED SOLUTION METHODOLODY}

\section{Solution Framework}

Figure 1 provides the flow chart of the proposed solution framework, which consists of three main components: an Initial Candidate Route Set Generation Procedure (ICRSGP) (Fan 2004, and Fan and Machemehl 2006a) that generates all feasible routes, incorporating practical bus transit industry guidelines using the Dijkstra's shortest path algorithm (Ahuja et al. 1993) and Yen's k-shortest path algorithm (Yen 1971); a Network Analysis Procedure (NAP) (Fan 2004, and Fan and Machemehl 2006a) that decides transit demand matrix, assigns transit trips (Han and Wilson 1982, and Shih et al. 1998), determines service frequencies and computes performance measures using a two-staged 
Binary Logit Model - Inversely Proportional Model (BLM-IPM) model (Fan 2004, and Fan and Machemehl 2006a); and a Metaheuristic Search Procedure (MSP) that combines the previous two parts, guides the candidate solution generation process and selects an optimal set of routes from the huge solution space (Fan 2004, and Fan and Machemehl 2006a, 2006b).

\section{Figure 1: Flow Chart of the Proposed Solution Methodology}

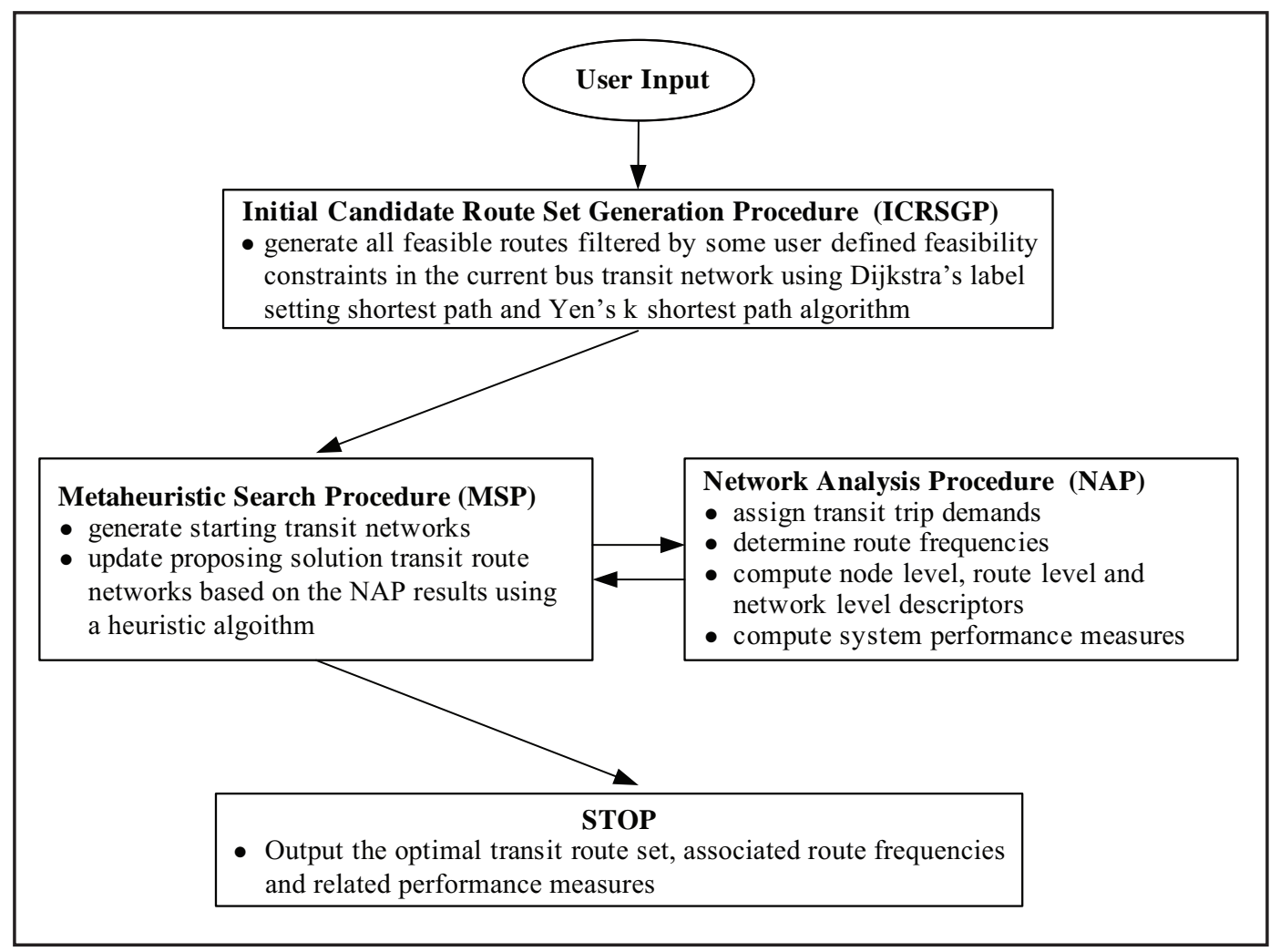

Figure 2 provides a flow chart of the NAP as a critical part of the BTRNDP with variable transit demand to evaluate the alternative network structure.

\section{Solution Algorithms}

As two of the most widely used metaheuristic methods to solve many combinatorial optimization problems, Genetic Algorithms (GA) (Fan 2004, Fan and Machemehl 2006a, Goldberg 1989, Holland 1975, and Michalewicz 1999) and Simulated Annealing (SA) (Fan and Machemehl 2006b, Koulamas et al. 1994, and Eglese 1990) are chosen to solve the BTRNDP. To examine the solution quality of the combined GA and SA and determine whether algorithm order matters, warm GA (using solutions obtained from SA as a starting point for each route set size) and warm SA (using solutions obtained from GA as a starting point for each route set size) are both proposed and implemented in this paper.

Figure 3 provides a flow chart of the GA-based solution approach for the BTRNDP with variable transit demand. Solution approach based on the SA can be seen from Fan 2004, and Fan and Machemehl 2006b. 
Bus Transit Route Network Design Problem

Figure 2: Network Analysis Procedure (NAP) for the BTRNDP

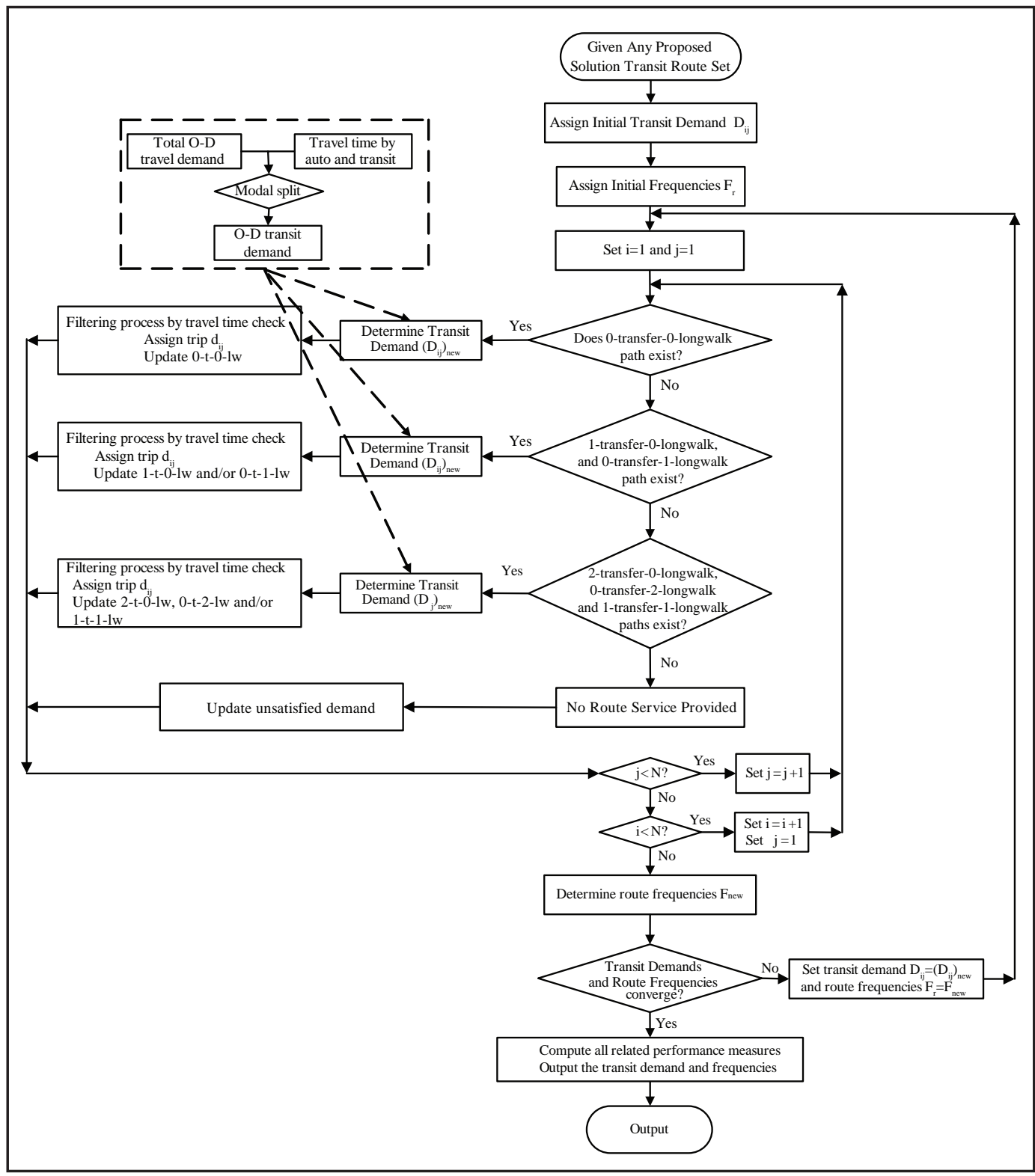

66 
Figure 3: A Genetic Algorithm-Based Solution Approach for the BTRNDP with Variable Transit Demand

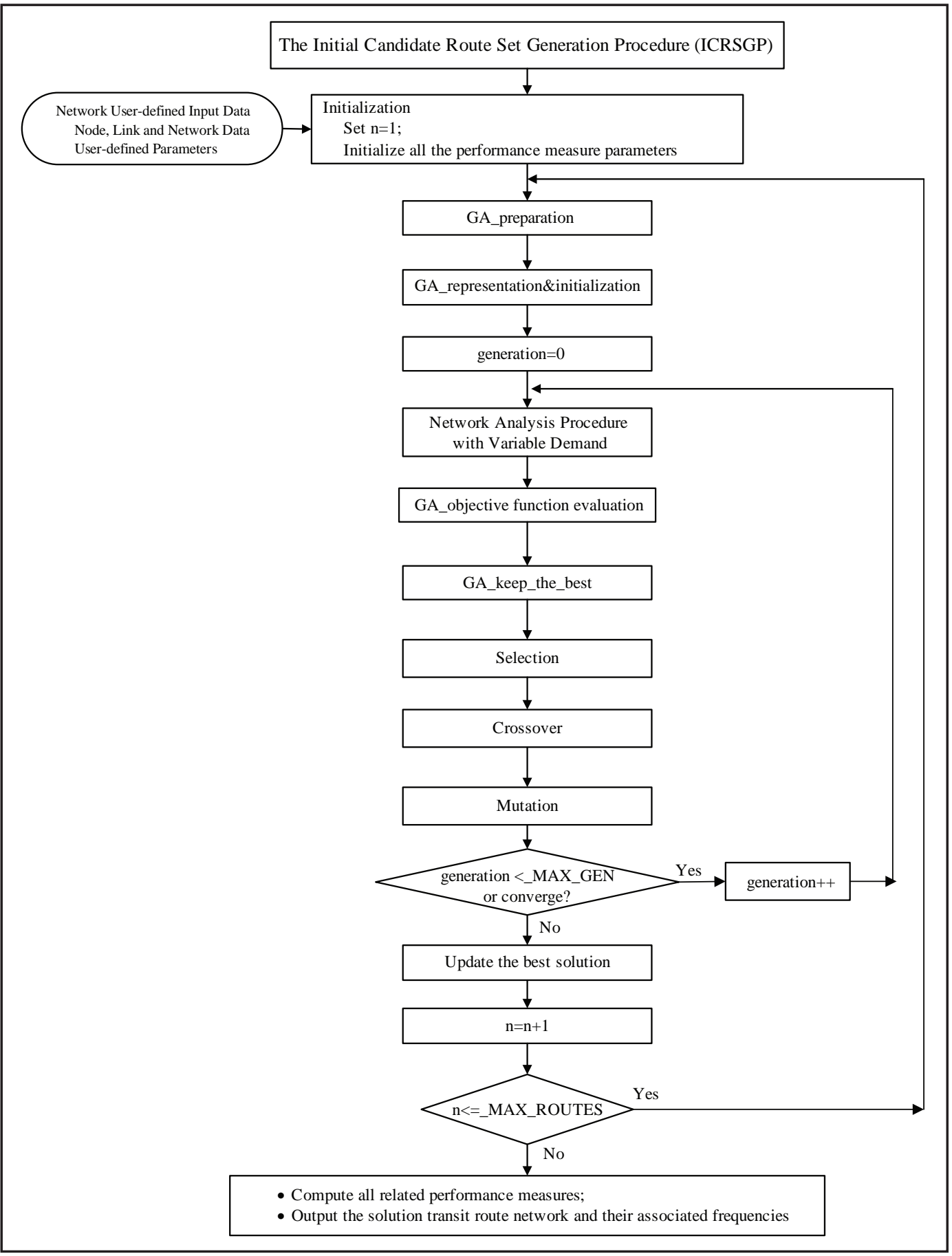




\section{EXPERIMENTAL NETWORK AND NUMERICAL RESULTS}

Several example networks are designed (Fan 2004) and the proposed algorithms implemented and tested using software developed in $\mathrm{C}++$.

\section{Example Network Configuration}

The GA/SA based solution methodology is implemented using the example network shown in Figure 4. This example network contains 28 travel demand zones and 65 road intersections. The BTRNDP zonal demands assume a distribution identical to the demand distribution of an auto-only network. The example network seeks to illustrate the proposed solution methodology for real-world applications. That is, for the large-scale BTRNDP, the zonal demands should be aggregated at the distribution node level in consideration of computation time, an assertion which is supported later in the computational necessity section. The chosen distribution node location might be obvious (only one entrance/exit for a particular travel zone) or less obvious (two or more locations where one could set the zonal demand aggregation point). After engaging in preliminary data processing, the example network contained 28 centroid distribution nodes, 93 nodes, and 284 arcs.

Figure 4: An Example Network with Graphical Representations for Nodes, Links and Routes

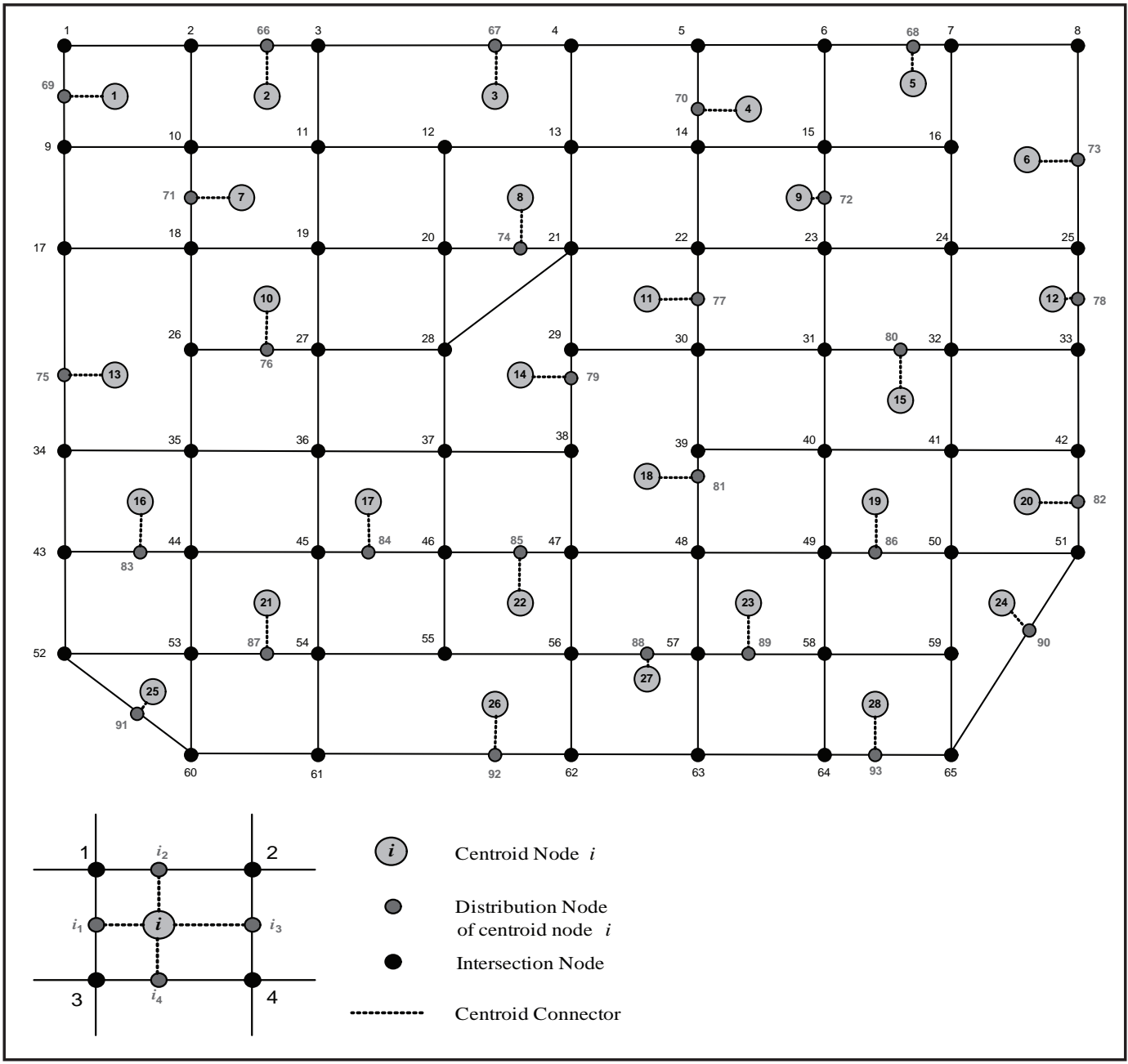




\section{Sensitivity Analyses}

The characteristics of the BTRNDP are extensive and complex due to its multi-objective decision making nature and the variety of parameters and procedures involved. The behavior of the BTRNDP depends upon the network size, the chosen parameters in the solution process and the chosen weight set level for each component of the objective function. In this sense, it is very hard to perform the sensitivity analysis in each case and generalize the behavior (e.g., the effect of route set size) of the BTRNDP. However, it is found that for the BTRNDP with variable transit demand, the characteristic behavior of the solutions obtained is similar in most cases and follows a somewhat predictable pattern (Fan 2004). In that regard, the numerical results based upon weights of 0.4, 0.2 and 0.4 for the user cost, operator cost and unsatisfied demand cost respectively, are chosen in this paper and used as a baseline for sensitivity analyses.

Table 1 presents a resulting set of optimal parameters derived from the sensitivity analyses of the GA and SA. Details on how to conduct the sensitivity analyses and how each set of optimal parameter values inherent in GA and SA are selected were presented in previous work (Fan 2004, and Fan and Machemehl 2006a, 2006b).

Table 1: Resulting Set of Optimal Parameters for the GA and SA

\begin{tabular}{lc|lc}
\hline \multicolumn{2}{c|}{ Genetic Algorithms } & \multicolumn{2}{c}{ Simulated Annealing } \\
\hline Population Size & 50 & Temperature & 2000 \\
Generations & 100 & Generations & 20 \\
Crossover Probability & 0.8 & Alpha Value & 0.6 \\
Mutation Probability & 0.1 & Repetition Counter & 10 \\
\hline
\end{tabular}

\section{Computational Necessity of Zonal Demand Aggregation}

The impacts of zonal demand aggregation on the computation time and solution quality are examined using the example network. A representative numerical result is given in Figure 5.1 to show the effects of zonal demand aggregation on solution quality for the BTRNDP, setting the weight of the unsatisfied demand cost as 0.4 and varying the weights of the user cost. As can be seen, the objective function values are smaller (more desirable) for the BTRNDP without zonal demand aggregation than with zonal demand aggregation at any weight set level. This is expected because the BTRNDP with zonal demand aggregation is an approximated (i.e., more constrained) version of the BTRNDP without zonal demand aggregation. The only difference is that the former (with zonal demand aggregation) assigns all zonal demands onto a chosen location rather than multiple distribution node locations. Therefore, the optimal objective function that can be achieved in the latter case is usually less than that in the former case (it will always be less if the BTRNDP is a convex rather than a non-convex problem).

If one tends to emphasize the solution quality and wants an optimal solution network, the BTRNDP without zonal demand aggregation is obviously the better choice. However, the improved solution quality from the BTRNDP without zonal demand aggregation comes at the cost of a significantly slower computation speed as shown in Figure 5.3. For example, the observed computing time for the BTRNDP with zonal demand aggregation using the example network is about three hours (the computation time for the GA and SA are almost the same) while that for the BTRNDP without zonal demand aggregation is about 72 hours. This is due to the combinatorial nature of the BTRNDP, in which the BTRNDP with zonal demand aggregation has a smaller solution space, less overlapping nodes among different routes, and much fewer combinations of routes than that without zonal demand aggregation. Also, since the BTRNDP with zonal demand aggregation has a smaller solution space, one can see from Figure 5.1 that the computational result is more consistent and has 
Figure 5: Effects of Zonal Demand Aggregation on Computation Performance and Efficiency for the BTRNDP

\section{Figure 5.1}

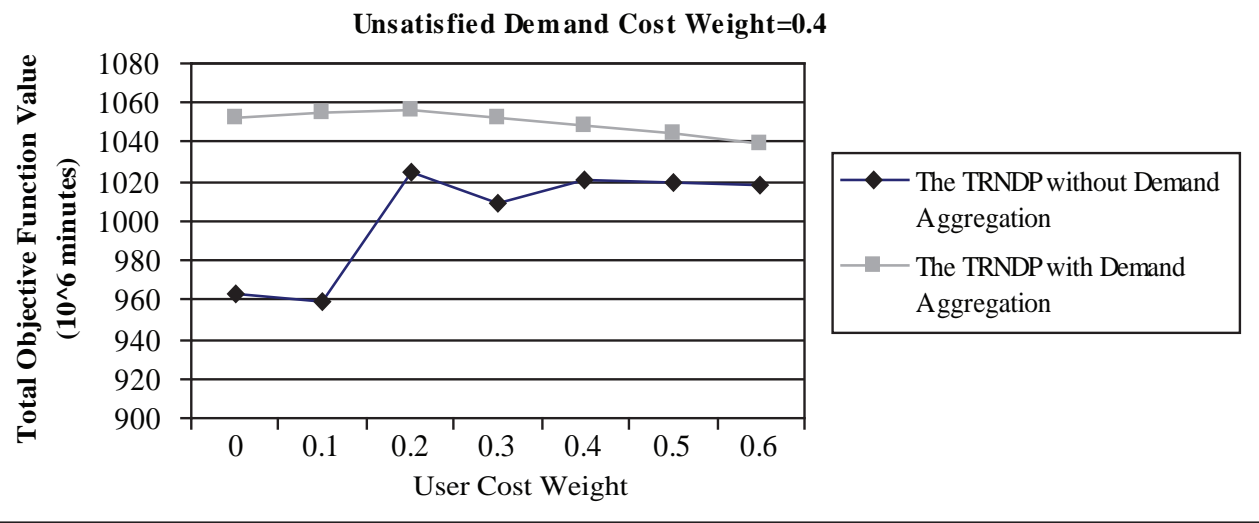

\section{Figure 5.2}

Unsatisfied Dem and C ost Weight $=0.4$

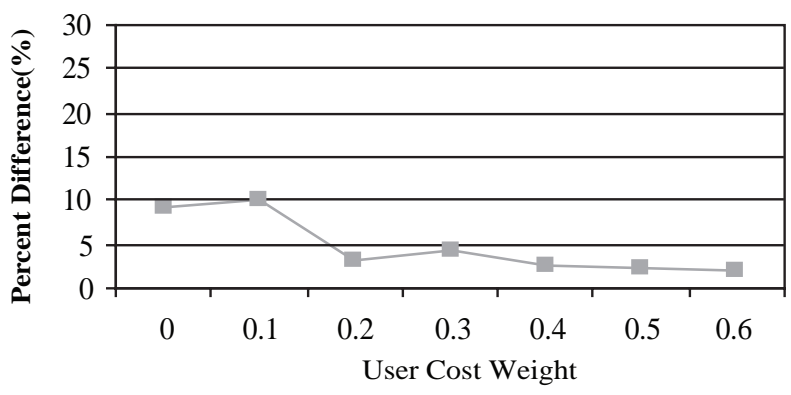

Percent Difference in

Objective Function $V$ alue

between with and without

Demand A ggregation (\%)

Figure 5.3

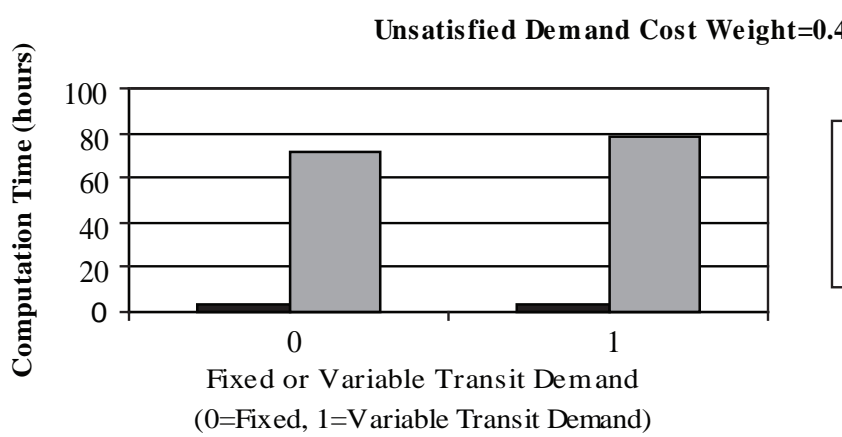

The TRNDP with Demand A ggregation

$\square$ The TRNDP without Demand A ggregation

less variation compared to that without zonal demand aggregation. The computer used for testing has a configuration of $\mathrm{P} 4 \mathrm{CPU} 2.0 \mathrm{GHz}$ and $512 \mathrm{MB}$ of memory.

Figure 5.2 further illustrates the relative difference in the objective function value between the BTRNDP with and without zonal demand aggregation. As one can see, the relative difference is not 
great (ranged from 2-10\%). Interestingly, on all conducted experiments, the lower the weight of the operator cost in the BTRNDP (worry less about the operator cost), the less the relative difference. This is probably attributable to the high penalty cost for the unsatisfied demand cost. With lower regard for operator cost and somewhat simplified solution space, the algorithms can focus more on finding a set of (different but similar in both scenarios) routes that may need large fleet size but provide service as good as possible to satisfy most transit users for the BTRNDP with zonal demand aggregation. These computational observations suggest the BTRNDP with zonal demand aggregation is highly relevant for real-world application.

\section{Computational Performance and Efficiency}

The computational performance analysis of the proposed metaheuristics is conducted through algorithm comparisons between GA alone, SA alone, warm GA (using solutions obtained from SA as a starting point for each route set size), and warm SA (using solutions obtained from GA as a starting point for each route set size) using the example network. Of further interest, pure random search (which randomly searches and selects route set within the solution space) is also used as a benchmark for solution quality comparison.

Figure 6 presents the algorithm solution quality comparisons for the BTRNDP. As expected, all metaheuristics are better than the pure random search method. It is very interesting to see that GA (alone) outperforms SA (alone) in nearly all cases of the BTRNDP with zonal demand aggregation. This is distinguished from previous conclusions that SA is better than GA for the BTRNDP without zonal demand aggregation (Fan 2004, and Fan and Machemehl 2006b). This can be explained as follows: without zonal demand aggregation, there are many feasible routes that can be generated from the shortest path and $k$-shortest path algorithm from the IGRSGP for the same origin and destination node pair. The routes form the neighborhood, and a very good one, as part of the optimal set of routes that can be selected and explored by a neighborhood-based metaheuristic algorithm such as SA.

\section{Figure 6. Algorithm Solution Quality Comparisons for the BTRNDP with Zonal Demand Aggregation}

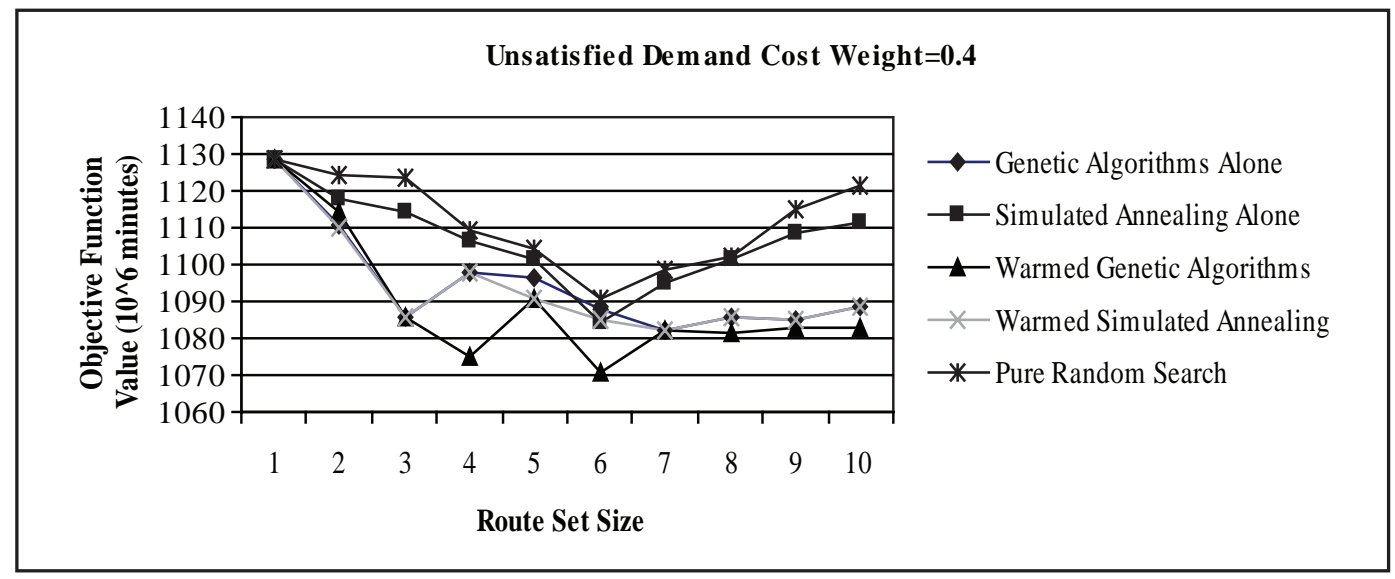

However, with zonal demand aggregation, there is a significant reduction in the amount of feasible routes for an origin - destination pair and therefore an increase in the possibility of eliminating some efficient routes for selection as part of optimal route set solution applying the neighborhood definition, resulting in poorer performance of SA. In the demand aggregated scenario, GA can perform better than the SA since it is not a neighborhood-based metaheuristic, rather it produces optimal solutions by selection, mutation and crossover. Also, the graph clearly shows that the warmed SA, which uses the solutions obtained from GA as a warm start, does not improve 
significantly in solution quality over the GA alone algorithm. However, the warmed GA, which uses the solutions obtained from SA as a warm start, is observed to be much better than the SA alone and actually outperforms all other algorithms for the BTRNDP. Based on the numerical results in Figure 6 , the SA, as a neighborhood based metaheuristic, may not be suitable for solving the real world BTRNDP. GA, one the other hand, can be used effectively to solve the large scale BTRNDP. Also, not all combined metaheuristics can be used to significantly improve the solution quality. Warm GA, which uses the solutions from the SA as a warm start, are highly recommended for the real world BTRNDP. In terms of computing efficiency, the computation time for the warm GA and warm SA are nearly the same and each takes about 4.5 hours using the same computer configuration.

\section{Effect of Route Set Size}

Figure 6 also displays the effect of the number of proposed routes in the transit network solution increasing from 1 to 10 . Based on these results, it is shown that as the route set size increases, the solution improves initially because more transit demand is assigned to the network and unsatisfied demand costs decrease. However, the lowest objective function value is achieved with six routes for the studied network and subsequent increases in the fleet size (i.e., operator costs) produce underutilization of routes and do not result in an improved objective function value.

\section{SUMMARY AND FUTURE RESEARCH}

The objective of this paper is to present computational insights on the optimal bus transit route network design problem (BTRNDP) with zonal demand aggregation and variable transit demand. A multi-objective nonlinear mixed integer model is developed. A general meta-heuristics based solution methodology is proposed. Genetic algorithms (GA), simulated annealing (SA), warmed GA, and warmed SA are implemented to solve the BTRNDP.

The necessity of zonal demand aggregation for the BTRNDP with variable transit demand is presented and justified. Algorithms are compared in terms of the solution quality. Computational results show that warmed GA outperforms all other tested algorithms and therefore is highly recommended for the large scale BTRNDP. Other characteristics, such as the effect of route set size are also presented.

Combining metaheuristic algorithms as an approach to solve the large scale BTRNDP is very promising. The performance of the combination of GA and SA with tabu search or other metaheuristic algorithms remains to be seen. Also, comparing solutions derived from these algorithms with those using existing transit route planning software such as EMME/2 and/or implantations using CPLEX may be also interesting. Future research may be directed toward these ends with further insight provided for solving large-scale instances of the BTRNDP.

\section{Acknowledgements}

The authors want to thank the U.S. Department of Transportation and the Southwest Region University Transportation Center for sponsoring this original research on the BTRNDP.

\section{References}

Ahuja, R.K., T.L. Magnanti, and J.B. Orlin. Network Flows: Theory, Algorithms and Applications. Prentice Hall, Englewood Cliffs, 1993.

Axhausemm K.W. and R.L. Smith. "Evaluation of Heuristic Transit Network Optimization Algorithms." Transportation Research Record 976, Journal of the Transportation Research Board, (1984): 7-20. 
Baaj, M.H. and H.S. Mahmassani. "An AI-Based Approach for Transit Route System Planning and Design.” Journal of Advanced Transportation 25(2), (1991): 187-210.

Bailey, L. "Public Transportation and Petroleum Savings in the U.S.: Reducing Dependence on Oil." ICF International, American Public Transportation Association, Washington, D.C., 2007.

Bielli, M., M. Caramia, and P. Carotenuto. "Genetic Algorithms in Bus Network Optimization." Transportation Research Part C 10, (2002): 19-34.

Ceder, A. and N.H.M. Wilson. "Bus Network Design." Transportation Research Part B 20(4), (1986): 331-344.

Ceder, A. and Y. Israeli. "User and Operator Perspective in Transit Network Design." Proc., The 77th Annual TRB Meeting, Paper No. 980267, Washington, D.C., 1998.

Chakroborty, P. "Genetic Algorithms for Optimal Urban Transit Network Design." Journal of Computer Aided Civil and Infrastructure Engineering 18, (2003): 184-200.

Chakroborty, P. and T. Dwivedi. “Optimal Route Network Design for Transit Systems using Genetic Algorithms.” Engineering Optimization 34(1), (2002): 83-100.

Chang, S.K. and P. Schonfeld. "Multiple Period Optimization of Bus Transit Systems.” Transportation Research Part B 25(6), (1991): 453-478.

Chang, S.K. and P. Schonfeld. "Welfare Maximization with Financial Constraints for Bus Transit Systems." Transportation Research Record 1395, (1993): 48-57.

Chien, S. and P. Schonfeld. "Optimization of Grid Transit System in Heterogeneous Urban Environments.” Journal of Transportation Engineering 123(1), (1997): 28-35.

Chien S., Z. Yang, and E. Hou. "A Genetic Algorithm Approach for Transit Route Planning and Design.” Journal of Transportation Engineering ASCE 127(3), (2001): 200-207.

Dubois, D., G. Bell, and M. Llibre. "A Set of Methods in Transportation Network Synthesis and Analysis.” Journal of Operations Research Society 30, (1979): 797-808.

Eglese, R.W. "Simulated Annealing: A Tool for Operational Research." European Journal of Operations Research 46, (1990): 271-281.

Fan, W. “Optimal Transit Route Network Design Problem: Algorithms, Implementations, and Numerical Results.” Dissertation (Ph.D.). The University of Texas at Austin, Austin, TX, 2004.

Fan, W. and R.B. Machemehl. "Optimal Transit Route Network Design Problem with Variable Transit Demand: A Genetic Algorithm Approach.” Journal of Transportation Engineering 132(1), (2006a): 40-51.

Fan, W. and R.B. Machemehl. "Using a Simulated Annealing Algorithm to Solve the Transit Route Network Design Problem.” Journal of Transportation Engineering 132(2), (2006b): 122-132.

Fan, W. and R.B. Machemehl. "A Tabu Search Based Heuristic Method for the Transit Route Network Design Problem." Proceedings of the $9^{\text {th }}$ International Conference on Computer-Aided Scheduling of Public Transport (CASPT), San Diego, CA, August 2004.

Goldberg, D.E. Genetic Algorithms in Search, Optimisation, and Machine Learning, AddisonWesley Reading, MA, London, 1989. 
Bus Transit Route Network Design Problem

Han, A. F. and N. Wilson. "The Allocation of Buses in Heavily Utilized Networks with Overlapping Routes.” Transportation Research Part B 16, (1982): 221-232.

Holland, J.H. Adaptation in Natural and Artificial Systems. The University of Michigan Press, Ann Arbor, MI, 1975.

Koulamas, C., S.R. Antony, and R. Jaen. "A Survey of Simulated Annealing Applications to Operations Research Problems.” Omega, 22(1), (1994): 41-56.

Lampkin, W. and P.D. Saalmans. "The Design of Routes, Service Frequencies and Schedules for a Municipal Bus Undertaking: A Case Study.” Operation Research Quarterly 18, (1967): 375-397.

LeBlanc, L.J. “Transit System Network Design.” Transportation Research Part B 22(5), (1988): 383-390.

Lee, Y.J. and V.R. Vuchic. "Transit Network Design with Variable Demand.” Proc., the $79^{\text {th }}$ Annual Meeting of TRB, Washington, D.C., 2000.

Michalewicz, Z. Genetic Algorithms + Data Structure = Evolution Programs, Third Edition, SpringerVerlag, New York, 1999.

NCHRP Synthesis of Highway Practice 69. Bus Route and Schedule Planning Guidelines, Transportation Research Board, National Research Council, Washington, D.C., 1980.

Newell, G.F. “Some Issues Relating to the Optimal Design of Bus Routes.” Transportation Science 13(1), (1979): 20-35.

Pattnaik, S.B., S. Mohan, and V.M. Tom. "Urban Bus Transit Network Design Using Genetic Algorithm.” Journal of Transportation Engineering 124(4), (1998): 368-375.

Rea, J.C. "Designing Urban Transit Systems: An Approach to the Route Technology Selection Problem.” PB 204881, University of Washington, Seattle, WA, 1971.

Shih, M.C., H.S. Mahmassani, and M.H. Baaj. "A Planning and Design Model for Transit Route Networks with Coordinated Operations." Proc. of the $77^{\text {th }}$ Annual Meeting of TRB, Paper No. 980418, Washington, D.C., 1998.

Shih, M., H.S. Mahmassani, and M.H. Baaj. “Trip Assignment Model for Timed-Transfer Transit Systems.” Transportation Research Record 1571, (1998): 24-30.

Silman, L.A., Z. Barzily, and U. Passy. "Planning the Route System for Urban Buses." Computers and Operations Research 1, (1974): 201-211.

Spasovic, L.N., M.P. Boilé, and A.K. Bladikas. "Bus Transit Service Coverage for Maximum Profit and Social Welfare.” Transportation Research Record 1451, (1994): 12-22.

Spasovic, L.N. and P. Schonfeld. "A Method for Optimizing Transit Service Coverage." Transportation Research Record 1402, (1993): 28-39.

Van Nes, R., R. Hamerslag, and B.H. Immer. "The Design of Public Transport Networks." Transportation Research Record 1202, (1988): 74-83.

Yen, J.Y. "Finding the K Shortest Loopless Paths in a Network." Management Science 17(11), (July, 1971): 712-716. 
Zhao, F. and X. Zeng, "Optimization of User and Operator Cost for Large Scale Transit Networks." Journal of Transportation Engineering 133(4), (2007): 240-251.

Dr. Wei (David) Fan is an assistant professor of civil engineering at The University of Texas at Tyler. He worked as a senior optimization developer in the R\&D department at SAS Institute Inc. after he obtained a Ph.D. degree in civil engineering from the University of Texas at Austin in 2004. His primary research interests include transportation network modeling \& optimization, traffic simulation and operations, and statistical analysis of transportation data. He has 14 years of experience with statistical and optimization computer software development, has authored more than 20 journal and conference publications, and has given numerous conference and invited presentations of his research results.

Dr. Randy Machemehl currently serves as a professor of civil engineering and directs the Center for Transportation Research at The University of Texas at Austin. He is an internationally recognized scholar in the transportation system engineering field and a well-known expert in several research fields including traffic control systems, simulation and optimization of transportation systems, psycho-physical measuring of driver behavior, and freeway bottleneck resolution. He has directed many large research projects and published more than 160 technical reports and papers.

Dr. Nicholas Lownes is an assistant professor in the Department of Civil and Environmental Engineering at the University of Connecticut. He specializes in traffic engineering \& operations, traffic simulation, and public transportation systems and has published in numerous journals and conferences and given many conference and invited presentations of his research results. 